enucleation is employed quite frequently even at institutions that do not support its ubiquitous use. These data lay the groundwork for determining whether RT is a modifiable variable for functional and oncologic outcomes in patients who undergo NSS.

\section{6 \\ ENDOSCOPIC ROBOT-ASSISTED SIMPLE ENUCLEATION (ERASE) VS. OPEN SIMPLE ENUCLEATION (OSE) FOR THE TREATMENT OF CLINICAL T1 RENAL MASSES: ANALYSIS OF PREDICTORS OF TRIFECTA OUTCOME}

Matteo Bonifazi, Andrea Mari, Francesco Sessa, Riccardo Campi, Tommasi Chini, Davide Vanacore, Riccardo Tellini, Mauro Gacci, Alberto Lapini, Lorenzo Masieri, Graziano Vignolini, Sergio Serni, Marco Carini and Andrea Minervini

Division of Urology, Department of Urology, Careggi Hospital, University of Florence, Florence (FI), Italy

Aim: The aim of this study was to analyse the intra- and postoperative complications, as well as the predictive factors of Trifecta outcome in patients submitted to endoscopic robotassisted simple enucleation (ERASE) and open simple enucleation (OSE) for clinical T1 renal masses. Materials and Methods: Overall, 634 cases treated with OSE $(\mathrm{n}=290)$ and ERASE $(n=344)$ were prospectively recorded in our Department between 2006 and 2014. Trifecta was defined as simultaneous ischemia time $<25 \mathrm{~min}$, no surgical complication and negative surgical margin. A univariate analysis and multivariate logistic regression were performed for Trifecta. Results: The two groups were comparable for body mass index (BMI), comorbidity, tumor side, clinical $\mathrm{T}$ score, tumor diameter, surgical indication, pre-operative renal function, pre-operative hemoglobin and hematocrit. A significant difference was found between the OSE and the ERASE groups in operative time (115 (96-130) vs. 150 (120180 ) minutes, $p<0.0001$ ), pedicle clamping (93.8\% vs. $69.2 \%, p<0.0001)$, estimated blood loss (EBL) (150 (100200) vs. $100(100-143) c c, p<0.0001)$ and intraoperative complications $(3.4 \%$ vs. $1.7 \%, p=0.02)$. The two groups were comparable for warm ischemia time (WIT) $\geq 25 \mathrm{~min}$. A significant difference was found between OSE and ERASE in overall $(16.6 \%$ vs. $5.5 \%, p<0.0001)$, Clavien $2(11.7 \%$ vs $4.4 \%, p=0.02)$ and Clavien $3(3.1 \%$ vs. $1.7 \%, p=0.04)$ postoperative surgical complications, length of stay (6.0 (5.0-7.0) vs. $5.0(4.0-6.0)$ days, $p<0.0001)$, pre-operative 1 st day delta creatinine $(0.3(0.2-0.4) v s .0 .15(0.1-0.2) \mathrm{mg} / \mathrm{dl}, p<0.0001)$, positive surgical margins $(2.1 \%$ vs. $1.5 \%, p=0.04)$, and Trifecta achievement $(73.8 \%$ vs. $85.5 \%, p<0.0001)$. At univariate analysis, a higher median clinical diameter, a higher mean age, a higher median Charlson comorbidity index (CCI), endophytic tumor growth pattern, renal sinus and calyceal dislocation of the tumor, a higher median PADUA score and OSE were predictive factors of Trifecta achievement. At multivariate analysis, CCI lost significance $(p=0.26)$, while age (odds ratio $(\mathrm{OR})=1.02,95 \%$ confidence interval $(95 \% \mathrm{CI})=1.00-1.04, p=0.001)$, clinical diameter $(\mathrm{OR}=1.22, \mathrm{CI}=1.05-1.42, p=0.008)$, PADUA score $(\mathrm{OR}=1.23$, $\mathrm{CI}=1.07-1.41, p=0.004)$ and $\mathrm{OSE}(\mathrm{OR}=1.74, \mathrm{CI}=1.13-2.68$, $p=0.01)$ were confirmed predictive factors for Trifecta failure. Conclusion: The ERASE is a feasible and safe technique, which shows a comparable WIT, together with a significantly lower EBL, surgical complications' rate, length of stay and a significantly higher Trifecta achievement compared to OSE. Age, comorbidity, tumor diameter and PADUA score, in association with surgical approach, represent significant predictive factors of Trifecta failure.

87

PROSPECTIVE ANALYSIS OF COMPLICATIONS
AND THEIR PREDICTIVE FACTORS AFTER
PARTIAL NEPHRECTOMY IN A MULTICENTER
COMPARATIVE ITALIAN STUDY (RECORD1)

Andrea Mari ${ }^{1}$, Andrea Minervini ${ }^{1}$, Alessandro Antonelli ${ }^{2}$, Riccardo Bertolo ${ }^{3}$, Giampaolo Bianchi ${ }^{4}$, Marco Borghesi ${ }^{5}$, Cristian Fiori $^{3}$, Nicola Longo ${ }^{6}$, Giuseppe Martorana ${ }^{5}$, Vincenzo Mirone ${ }^{6}$, Giuseppe Morgia ${ }^{7}$, Giacomo Novara ${ }^{8}$, Francesco Porpiglia ${ }^{9}$, Bruno Rovereto ${ }^{10}$, Riccardo Schiavina ${ }^{5}$, Sergio Serni ${ }^{1}$, Francesco Sessa ${ }^{1}$, Claudio Simeone ${ }^{2}$, Mario Sodano ${ }^{2}$, Riccardo Tellini ${ }^{1}$, Carlo Terrone ${ }^{11}$ and Marco Carini ${ }^{1}$

${ }^{1}$ Division of Urology, Department of Urology, Careggi Hospital, University of Florence, Florence, Italy;

${ }^{2}$ Department of Urology; Azienda AO Spedali

Civili Di Brescia, Italy;

${ }^{3}$ Department of Urology, University of Turin, Ospedale San Luigi Gonzaga, Orbassano, Italy;

${ }^{4}$ Policlinico Di Modena, Department of Urology,

University of Modena, Italy;

${ }^{5}$ Department of Urology, University of Bologna,

S. Orsola-Malpighi Hospital, Bologna, Italy;

${ }^{6}$ Urology Department, University Federico II of Naples, Via S.Pansini, Naples, Italy;

${ }^{7}$ Luna Foundation, Roma, Italy;

${ }^{8}$ Department of Urology, University of Padua, Padua, Italy; ${ }^{9}$ Department of Urology, University of Turin,

Ospedale San Luigi Gonzaga, Orbassano, Italy;

${ }^{10}$ I.R.C.C.S. Policlinico San Matteo,

Department of Urology, Pavia, Italy;

${ }^{11}$ Urology, Maggiore Della Carità University Hospital, University of Eastern Piedmont, Novara, Italy 